\title{
Cortical Neural Activity Predicts Sensory Acuity Under Optogenetic Manipulation
}

\author{
ㄱohn J. Briguglio, ${ }^{1,2,3}$ @Mark Aizenberg, ${ }^{2}$ Vijay Balasubramanian, ${ }^{1}$ and $\oplus^{-M a r i a ~ N . ~ G e f f e n ~}{ }^{2}$ \\ ${ }^{1}$ Department of Physics, Department of Otorhinolaryngology HNS, Department of Neuroscience, University of Pennsylvania, Philadelphia, Pennsylvania \\ 19104, ${ }^{2}$ Department of Otorhinolaryngology-Head and Neck Surgery, Department of Neuroscience, University of Pennsylvania, Philadelphia, Pennsylvania \\ 19104, and ${ }^{3}$ Janelia Research Campus, Ashburn, Virginia 20147
}

Excitatory and inhibitory neurons in the mammalian sensory cortex form interconnected circuits that control cortical stimulus selectivity and sensory acuity. Theoretical studies have predicted that suppression of inhibition in such excitatory-inhibitory networks can lead to either an increase or, paradoxically, a decrease in excitatory neuronal firing, with consequent effects on stimulus selectivity. We tested whether modulation of inhibition or excitation in the auditory cortex of male mice could evoke such a variety of effects in tone-evoked responses and in behavioral frequency discrimination acuity. We found that, indeed, the effects of optogenetic manipulation on stimulus selectivity and behavior varied in both magnitude and sign across subjects, possibly reflecting differences in circuitry or expression of optogenetic factors. Changes in neural population responses consistently predicted behavioral changes for individuals separately, including improvement and impairment in acuity. This correlation between cortical and behavioral change demonstrates that, despite the complex and varied effects that these manipulations can have on neuronal dynamics, the resulting changes in cortical activity account for accompanying changes in behavioral acuity.

Key words: auditory cortex; behavior; computational modeling; excitatory-inhibitory circuits; frequency discrimination; optogenetics

\section{Significance Statement}

Excitatory and inhibitory interactions determine stimulus specificity and tuning in sensory cortex, thereby controlling perceptual discrimination acuity. Modeling has predicted that suppressing the activity of inhibitory neurons can lead to increased or, paradoxically, decreased excitatory activity depending on the architecture of the network. Here, we capitalized on differences between subjects to test whether suppressing/activating inhibition and excitation can in fact exhibit such paradoxical effects for both stimulus sensitivity and behavioral discriminability. Indeed, the same optogenetic manipulation in the auditory cortex of different mice could improve or impair frequency discrimination acuity, predictable from the effects on cortical responses to tones. The same manipulations sometimes produced opposite changes in the behavior of different individuals, supporting theoretical predictions for inhibition-stabilized networks.

\section{Introduction}

Sensitivity to sensory signals depends on neuronal tuning to specific parameters of sensory stimuli, such as orientation of edges

\footnotetext{
Received Aug. 28, 2017; revised Nov. 14, 2017; accepted Dec. 15, 2017.

Author contributions: J.J.B., M.A., V.B., and M.N.G. designed research; J.J.B., M.A., and M.N.G. performed research; J.J.B., M.A., V.B., and M.N.G. contributed unpublished reagents/analytic tools; J.J.B., M.A., and M.N.G. analyzed data; J.J.B., M.A., V.B., and M.N.G. wrote the paper.

This work was supported by the National Institutes of Health (Grants R03DC013660, R01DC014700, and R01DC015527 to M.N.G.), a Klingenstein Award in Neuroscience to M.N.G., a Human Frontier in Science Foundation Young Investigator Award to M.N.G., a Pennsylvania Lions Club Hearing Research Fellowship (M.G.N.) and the National Science Foundation (Grant PHY1058202 to V.B.). M.N.G. is the recipient of a Burroughs Wellcome Award at the Scientific Interface. We thank the members of the Geffen laboratory and the Hearing Research Center at the University of Pennsylvania, including Steve Eliades, Yale Cohen, Katherine Wood, Jennifer Blackwell, Ryan Natan, and Christopher Angeloni, for helpful discussions.

The authors declare no competing financial interests.

Correspondence should be addressed to Maria N. Geffen, Department of Otorhinolaryngology HNS, University of Pennsylvania, 5 Ravdin, 3600 Spruce Street, Philadelphia, PA 19104. E-mail: mgeffen@pennmedicine.upenn.edu.
}

for visual stimuli or tone frequency for auditory stimuli. Such neuronal selectivity arises in many brain areas and is shaped by complex, interconnected circuits of excitatory and inhibitory neurons (Isaacson and Scanziani, 2011). The balance between inhibitory and excitatory stimulus representation in the sensory cortex has been proposed to underlie learning- and adaptationdependent changes in stimulus-driven responses (Froemke, 2015). Recently, a number of studies have begun to unravel the role of inhibition in sensory processing, empowered by recently developed optogenetic techniques (Lee et al., 2012; Wilson et al., 2012; Atallah et al., 2012; Aizenberg et al., 2015; Marlin et al., 2015; Natan et al., 2015; Seybold et al., 2015; Phillips and Hasenstaub, 2016). These methods drive specific classes of neurons to express an opsin such 
that shining light over that brain area either activates or suppresses neuronal activity selectively (Boyden et al., 2005; Zhang et al., 2007; Sohal et al., 2009; Cardin et al., 2010; Deisseroth, 2011).

Whereas optogenetic techniques provide exquisite molecular and temporal specificity for testing the function of specific cell types in sensory acuity, they currently carry inherent and technical limitations that can lead to different levels of opsin expression and activation or suppression strength across individual animals. Because measurements are typically performed in multiple animals, these specific differences are accounted for using statistical analyses while summarizing the average effects of optogenetic perturbations. We postulated that these differences could be instead exploited to characterize the diversity of effects across subjects, thereby deepening our understanding of the function of excitatory-inhibitory interactions in sensory processing.

It has been predicted theoretically that, within balanced excitatory-inhibitory circuits, increasing inhibition can either decrease excitatory neuronal activity or, paradoxically, increase it depending on specific perturbation parameters and circuit properties (Tsodyks et al., 1997). We therefore hypothesized that the differences in technical parameters of optogenetic stimulations across animals could evoke both positive and negative effects on the firing rate of the target neuronal population. Focusing on tone frequency representation in the auditory cortex (Aizenberg et al., 2015), we tested whether this was indeed the case by upregulating or downregulating the activity of either inhibitory or excitatory neurons and measuring the resulting changes in frequency discriminability based on neuronal population activity. Our previous analyses had demonstrated that, on average, suppressing the activity of the most common class of interneuron, parvalbumin-positive neurons (PVs), in auditory cortex (AC) impaired behavioral frequency discrimination acuity, whereas activating PVs improved it; activating excitatory neurons did not have an effect (Aizenberg et al., 2015). Here, we compared changes in neuronally predicted and behavioral frequency discrimination acuity due to optogenetic manipulations.

Optogenetic interventions led to changes in tone-evoked responses of recorded neurons. As predicted, the same manipulation sometimes produced opposite changes in neurometric sensitivity and in behavior for different individuals. Computational analysis predicted consequent changes in discrimination thresholds, which explained the measured behavioral changes, including improvement or impairment of discrimination driven by the same optogenetic manipulation in different individuals. Our results thus demonstrate that, although these manipulations have complex effects on the neural network, the resultant changes in activity are sufficient to predict changes in behavior.

\section{Materials and Methods}

Animals. All experiments were performed in adult male mice (The Jackson Laboratory; age 12-15 weeks; weight 22-32 g; PV-Cre mice, strain: B6; 129P2-Pvalbtm1(cre)Arbr/J; CamKII $\alpha$-Cre: B6.Cg-Tg(CamKII $\alpha$ Cre)T29-1Stl/J) housed at $28^{\circ} \mathrm{C}$ on a $12 \mathrm{~h}$ light/dark cycle with water and food provided ad libitum with five or fewer animals per cage. Note that B6 (C57BL/6) mice exhibit accelerated hearing loss, but are expected to exhibit normal hearing in the age range used at the frequencies and sound pressure levels used in the study (Mikaelian, 1979; Erway et al., 1993; Johnson et al., 2017). All animal work was conducted according to the guidelines of University of Pennsylvania Institutional Animal Care and Use Committee and the Association for Assessment and Accreditation of Laboratory Animal Care International guide on animal research. Anesthesia by isofluorane and euthanasia by carbon dioxide were used. All means were taken to minimize the pain or discomfort of the animals during and after the experiments. The data were collected in the same set of experiments as reported previously (Aizenberg et al., 2015). This report uses data only from those mice, in which we recorded from at least $n=10$ frequency tuned neurons.

Viral constructs. Modified adeno-associated virus (AAV) vectors were obtained from the Penn VectorCore. To suppress PVs, we used modified AAV vector encoding Archaerhodopsin (Arch) under FLEX design (Addgene plasmid 22222, AAV-FLEX-Arch-GFP (Chow et al., 2010). To activate either PVs in PV-Cre mice and or excitatory neurons in CamKII $\alpha$-Cre mice, we used modified AAV encoding Channelrhodopsin (ChR2) under FLEX design (Addgene plasmid 18917 AAV-FLEX-ChR2- tdTomato, ChR2 (Atasoy et al., 2008). As a control, we used modified AAV vectors encoding only green fluorescent protein (GFP) or tdTomato under FLEX design.

Experimental methods overview. The methods used have been described previously (Aizenberg et al., 2015). Briefly, at least $10 \mathrm{~d}$ before the start of experiments, mice were injected with a viral construct, if any, and implanted with optical cannulas and a head post, as described previously, under isoflurane anesthesia. Viral construct injection targeted AC using stereotaxic map. Fiber-optic cannulas were implanted bilaterally over the injection site at depth of $0.5 \mathrm{~mm}$ from the scull surface along the dorsalventral axis. After recovery, mice were habituated to the head-fixing apparatus and subjected to behavioral frequency discrimination tests for 1-3 d, followed by electrophysiological recordings in the auditory cortex. On half of the trials in behavioral and neurophysiological recordings, light was presented through the fiber-optic cannula to activate or suppress target neurons. Upon conclusion of experiments, brains were extracted, fixed, and subjected to immunostaining. Viral spread was confirmed postmortem by visualization of the fluorescent protein expression in fixed brain tissue and its colocalization with PV or excitatory neurons after immunohistochemical processing with the appropriate antibody.

Behavioral frequency discrimination. Behavioral frequency discrimination was measured using a modified prepulse inhibition (PPI) of the acoustic startle reflex procedure on a daily basis (Clause et al., 2011; Aizenberg et al., 2015). As reported previously (Aizenberg and Geffen, 2013; Aizenberg et al., 2015), PPI provides psychometric response curves for frequency discrimination over the course of a single session that lasts $<1 \mathrm{~h}$ and does not require training the subject, which can confound interpretation of fear conditioning. Mice were head-fixed, connected to optical cannulas as needed, and placed on a load-bearing platform. Sound was presented through a speaker, consisting of a background tone (15 kHz, 10-20 s, $80 \mathrm{~dB}$ SPL) that, on each trial, switched to a prepulse tone $(10.2,12.6,13.8,14.7$, and $15.0 \mathrm{kHz}, 60 \mathrm{~ms}, 80 \mathrm{~dB}$ SPL) followed by startle noise (SS, broad-band noise, $100 \mathrm{~dB}$ SPL, $20 \mathrm{~ms}$ ). The frequency difference between the background and prepulse tone is denoted $\Delta f$. Each session started with presentation of 10 startle-only trials to ensure that the mouse habituated to the beginning of the session. Data from these trials were not used for the analysis. Each prepulse tone was repeated in a pseudorandom order at least five times during each behavioral session.

The acoustic startle response (ASR) for a given $\Delta f$ was computed as the average over trials of the difference between the maximum vertical force applied within the $500 \mathrm{~ms}$ window after SS and the average baseline activity during $500 \mathrm{~ms}$ before SS. In each PPI session, the PPI was calculated as follows:

$$
\operatorname{PPI}(\Delta f)=100 \frac{\operatorname{ASR}(0)-A S R(\Delta f)}{A S R(0)}
$$

where PPI is reported in percentage relative to maximum PPI and ASR $(\Delta f)$ is measured using the $50 \%$ of the strongest ASR magnitudes for each PP frequency. Fifty percent was chosen for consistency with previous work because the psychometric curve has the steepest slope at this value (Aizenberg and Geffen, 2013). Behavioral threshold was determined by fitting the PPI with a generalized logistic function and defining the threshold for the fit as the $\Delta f$ that produced $50 \%$ of the maximum PPI. The distance between the animal's frequency discrimination threshold and measured tone frequencies differed among subjects due to limited sampling of tone frequencies. Therefore, we computed a set of thresholds resulting from all fits that produced less than a $25 \%$ increase in mean squared error relative to the best fit (although increasing this cutoff to 
$60 \%$ yielded small differences). We took the mean and standard deviation (SD) of the resulting set of thresholds as the estimate of the subject's performance. Each psychometric function consisted of five data points representing the difference between the background frequency and five prepulse frequencies (PP). Each data point was obtained by averaging ASRs from all repetitions corresponding to a given frequency. In a standard PPI session, 20 repetitions of each PP were presented (100 trials in total). However, if either threshold was out of the range $(0.5-32 \%)$ or the fit coefficient of the curve $\left(R^{2}\right)$ was below 0.7 , then the mouse underwent an additional 10 repetitions (50 trials). If threshold and fit curve failed to meet the above criteria after 200 trials, the session was excluded from statistical analysis (three of 61 sessions). Previous studies have shown that psychometric thresholds obtained from day-to-day measurements were stable (Aizenberg and Geffen, 2013; Aizenberg et al., 2015; Mwilambwe-Tshilobo et al., 2015). Light-on trials included a $1 \mathrm{~s}$ laser presentation that starts $0.5 \mathrm{~s}$ preceding PP onset. Light-off trials included laser presentation at quasirandom position during the intertrial interval. All analysis was performed separately on light-on and light-off trials. Simple randomization was used to assign the subjects to the experimental groups. A pseudorandom sequence was used for tone presentation during PPI tests. During PPI procedure, the timing of the laser presentation on laser-off trials was pseudorandomized with respect to the timing of the tones. Blinding of the experiment with respect to animal groups was not possible because animals in different groups underwent different experimental protocols.

Neuronal tone response measurement. All recordings were performed inside a double-walled acoustic isolation booth (Industrial Acoustics) as described previously (Aizenberg et al., 2015). Activity of neurons in the primary auditory cortex of head-fixed, awake mice was recorded via a silicon multichannel probe (Neuronexus). Putative principal (excitatory) neurons were identified using waveform and spontaneous firing rate (for details, see Aizenberg et al., 2015). Acoustic stimulus was delivered via a calibrated magnetic speaker (Tucker-Davis Technologies) (Carruthers et al., 2013). The frequency tuning curves were measured by presenting a train of 50 pure tones (50 ms long, ISI $450 \mathrm{~ms}$ ) with frequencies spaced logarithmically between 1 and $80 \mathrm{kHz}$ and at 8 intensities (sound pressure levels, SPLs) spaced uniformly between 10 and $80 \mathrm{~dB}$, a standard procedure in characterizing auditory responses and determining the threshold amplitude for tone at each frequency for each neuron (Carruthers et al., 2013; Aizenberg et al., 2015). For data analysis, we averaged responses of the neurons to each tone across 3 highest amplitudes. Each tone was repeated twice in pseudorandom sequence and the stimulus was counterbalanced for laser presentation. On light-on trials, light was presented via the optic cannulas with an onset of $100 \mathrm{~ms}$ before tone onset and lasting for $250 \mathrm{~ms}$. The full stimulus was repeated five times.

Histology. Virus spread was confirmed postmortem by visualization of the fluorescent protein expression in fixed brain tissue and its colocalization with PV or excitatory neurons after immunohistochemical processing with the appropriate antibody.

Identification of putative excitatory neurons. Putative principal (excitatory) neurons were identified using waveform and spontaneous firing rate (for details, see Aizenberg et al., 2015).

Neural response analysis. The neural frequency response function was calculated using the average frequency tuning curve across the three highest intensities. The results were modeled using a Gaussian in logfrequency space as follows:

$$
F R(f)=B+A^{*} \exp \left[-\frac{\left(f-f_{0}\right)^{2}}{2 \sigma^{2}}\right]
$$

where $B$ is the baseline response, $A$ is the amplitude of the strongest evoked response (relative to baseline), $f_{0}$ is the frequency evoking the strongest response, and $\sigma$ is the width of the frequency response function. Only neurons with a Gaussian fit of $R^{2}>0.6$ were kept for further analysis.

To calculate the Fano factor of a neuron, we calculated the mean and variance of the firing rate to each combination of frequency and SPL. The slope of these data was taken as an estimate of the Fano factor.
The correlation between neurons (calculated only between simultaneously recorded neurons) was computed using a reduced measure of deviation from the mean for each neuron as follows:

$$
s_{i}^{k}(f, d)=\frac{r_{i}^{k}(f, d)-\bar{r}_{i}(f, d)}{\sqrt{F_{i} \bar{r}_{i}(f, d)}}
$$

where $k$ is the repetition number $(1-5), i$ is the neuron, $f$ is the frequency, $d$ is the intensity, $r$ is the evoked response, $\bar{r}$ is the average response (firing rate) of a neuron to a particular frequency and intensity, and $F$ is the Fano factor of that neuron. For a generalized Poisson process, $s_{\mathrm{i}}$ has zero mean and unit variance because the variance is proportional to the mean. The correlation between two neurons is then given by the following:

$$
C_{i, j}=s_{i}^{k}(f, d) s_{j}^{k}(f, d)_{k, f, d}
$$

Computing Fisher information. Fisher information was calculated to provide a measure of neurometric frequency discrimination. Fisher information was calculated numerically from the recorded data based on the characterization of the neural responses (see "Neural response analysis" section) and is given by the following:

$$
I_{F}(f)=\sum_{\bar{n}} P(\bar{n} \mid f)\left(\frac{\delta}{\delta f} \log P(\vec{n} \mid f)\right)^{2}
$$

where $P$ is the probability that the population of neurons produces $\vec{n}=$ $\left(n_{1}, n_{2}, \ldots\right)$ spikes in response to the tone $f$. Assuming Poisson variability and independent neurons, then:

$$
P(\vec{n} \mid f)=\prod_{i} P\left(n_{i} \mid f\right)=\prod_{i} \frac{e^{-\mu_{i}(f)} \mu(f)^{n_{i}}}{n_{i} !}
$$

where the first equation expresses the independent neuron assumption and the second step uses the assumption of Poisson variability. Here, $\mu_{\mathrm{i}}$ and $n_{\mathrm{i}}$ are the expected and observed number of spikes from neuron $i$, respectively. For mice in which multiple recording sessions were performed, neurons were pooled across sessions. A second model assumes independent neurons and uses a generalized Poisson distribution as follows:

$$
P\left(n_{i} \mid f\right)=\frac{\alpha_{i}(f)\left(\alpha_{i}(f)+n_{i} \lambda_{i}\right)^{n-1} e^{-\left(\alpha_{i}(f)+n_{i} \lambda_{i}\right)}}{n_{i} !}
$$

where $\alpha_{i}=\mu_{i}(f)^{\star} F_{i}^{-1 / 2}$ and $\lambda_{i}=1-F_{i}^{-1 / 2}$. This model only allows for Fano factors above 1, which is consistent with other models of neural variability and measured cortical variability (Goris et al., 2014; Blackwell et al., 2016). We therefore set any measured Fano factor $<1$ to exactly 1 for the purpose of the Fisher information calculation.

Estimating the number of neurons for neurometric thresholds. Animals displayed varying levels of frequency discrimination acuity and we measured different sized subsets of frequency-tuned neurons in each experiment. To estimate the number of neurons required to account for behavioral discrimination acuity from the measured population, we used the fact that Fisher information for a population of independent neurons is the sum of the Fisher information from each neuron.

$$
I^{(t o t a l)}=\sum_{n} I^{(n)}
$$

We then assume that the population of neurons that we measured are representative of the overall population (at least the population that contributes to discrimination at the relevant frequency) as follows:

$$
I^{(n)} \approx \frac{1}{N_{E}} \sum_{k=1}^{N_{E}} I_{E}^{(k)}=\frac{1}{N_{E}} I_{E}^{(\text {total })}
$$

Here we use $n$ to refer to the overall population that we seek to estimate, $k$ to index the neurons that were measured, $N_{\mathrm{E}}$ is the number of experimentally measured neurons that are frequency tuned, $I_{E}^{(k)}$ is the Fisher information of these experimentally measured neurons, and $I_{E}^{(t o t a l)}$ is the 

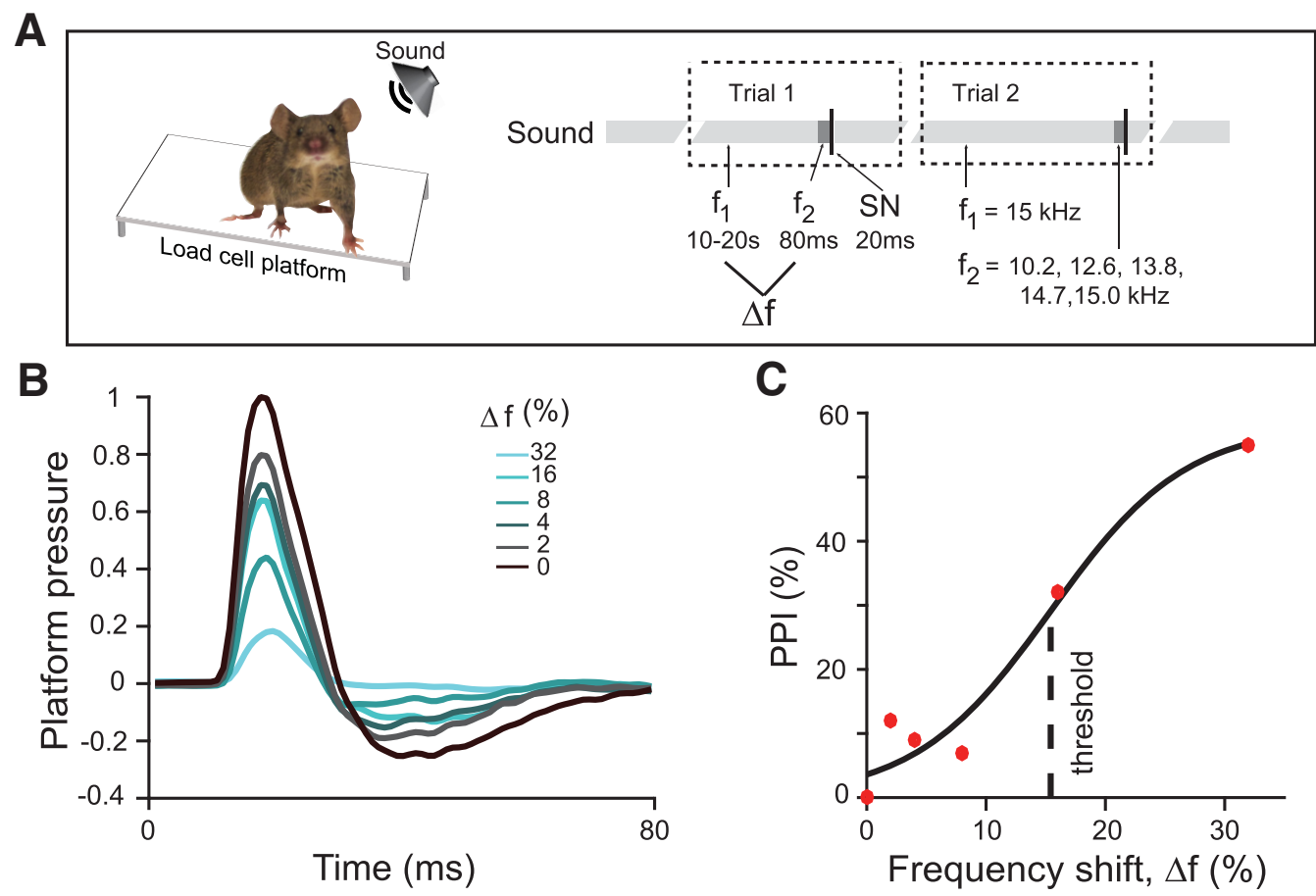

Figure 1. Measurement of behavioral frequency discrimination acuity. $A$, Schematic of measurement of frequency discrimination acuity in mouse. Left, Startle response measured as pressure the subject exerts on a platform. Right, Sound stimulus time course: an ongoing background tone (light gray, $f_{1}$ ) is followed by a brief prepulse tone of different frequency (dark gray band, $f_{2}$ ) and then by a startle noise (thin black band, SN). $\boldsymbol{B}$, Normalized time course of platform pressure during the startle response to noise for different prepulse tones for an exemplar mouse. Time relative to SN onset. C, PPI measured as reduction in the acoustic startle response as a function of the frequency shift $(\Delta f)$ between the background and prepulse tones (see Materials and Methods, Eq. 1) of an exemplar mouse. PPI does not reach $100 \%$ because, even with an easily identifiable prepulse tones, the animal still startles. Dots, Data; solid line, fit.

total information used to calculate the neurometric threshold. Recalling that the neurometric threshold is defined as $I^{-1 / 2}$, we then have the following:

$$
I^{(\text {total })}=\frac{N_{T}}{N_{E}} I_{E}^{(t o t a l)}
$$

where $N_{\mathrm{T}}$ is the number of neurons used in prediction of neurometric threshold. We can rewrite this as follows:

$$
N_{T}=N_{E}\left(\frac{t_{b e h}}{t_{\text {neu }}}\right)^{2}
$$

where $t_{\text {beh }}$ is this behaviorally measured threshold and $t_{\text {neu }}$ is the neurometric estimate of the threshold. This is plotted for each animal used in our analyses in Figure 7 and we find that the average for both light-on and light-off conditions is just about $10^{3}$ neurons. Uncertainty in these predictions was estimated by selecting a random subset of $90 \%$ of the experimentally measured neurons, computing the Fisher information, and taking the SD resulting from performing this 1000 times.

Comparing behavioral and neurometric thresholds. Neural responses were accumulated over all recording sessions for each mouse and used to compute the Fisher information for the animal. The Fisher information provides a bound on the variability of an unbiased estimator and any criterion level of decoding performance scales with the inverse square root of the Fisher information. If neural responses are independent, then the Fisher information scales linearly with the number of neurons. To compare threshold predictions between mice with different numbers of measured neurons, we computed the average Fisher information per neuron. This allowed us to compare across mice and to estimate the minimum number of effective frequency-tuned neural units that must contribute to explain the observed frequency discrimination performance (Cover and Thomas, 1991). To estimate the neurometric threshold, we first computed the inverse square root of the Fisher information per neuron and took an average over a small region around $15 \mathrm{kHz}$ (the baseline frequency used in behavioral tests). Only mice with $>10$ re- corded frequency-tuned neurons were included in the analysis (19 mice). Our estimate is a lower limit on the uncertainty in the estimate of this tone frequency based on the recorded population responses. Because Fisher information scales linearly in an independent population, this method also provides an estimate on the effective number of neurons that must contribute to the tone representation to account for behavioral discriminability (see Fig. 5). The observed neurometric discriminability with order 1000 independent neurons is similar to behavioral discrimination acuity.

Data availability. All relevant data will be deposited in the Dryad database and made publically available.

Code availability. All relevant code will be posted on Github and made publically available.

\section{Results}

\section{Measuring frequency discrimination acuity}

To measure frequency discrimination acuity, we used a procedure based on the PPI of the acoustic startle reflex (Fig. 1A,B). Like other mammals, mice startle to loud noise. The startle response, as measured by the change in pressure exerted by the subject on a balance platform, is typically decreased when the startle noise is preceded by a brief tone that mice can detect, a phenomenon termed PPI. We presented mice with a continuous tone at one frequency, which was stepped to a tone of a different frequency just before the startle noise. The startle response was attenuated as the frequency difference between background and prepulse tones increased (Fig. $1 B, C$ ). This attenuation reflects the ability of the mouse to detect frequency differences (Aizenberg and Geffen, 2013; Aizenberg et al., 2015). We characterized frequency discrimination acuity in terms of a behavioral threshold, the frequency difference that produced $50 \%$ of the maximum PPI (Fig. 1C). 

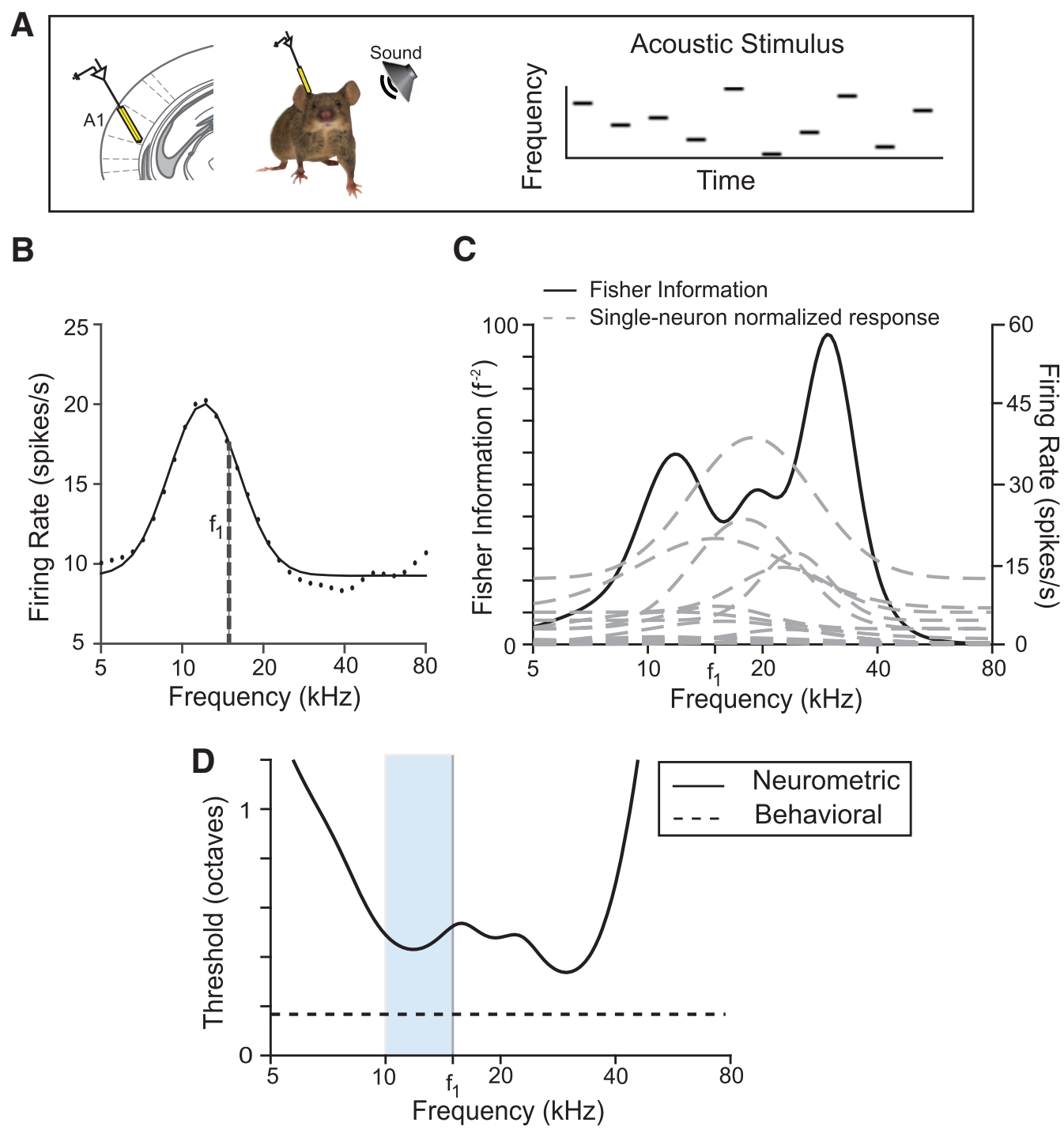

Figure 2. Measurement of neurometric frequency discrimination acuity. $\boldsymbol{A}$, Left, Schematic of electrophysiological recording of neuronal responses in the primary auditory cortex (A1) in awake mouse. Right, Stimulus consisting of a pseudorandom sequence of pure tones at varying frequency and intensity levels. $\boldsymbol{B}$, Representative frequency response function for a single neuron $\left(f_{1}=\right.$ background tone in Fig. 1). Black dots, Data; black line, fit. C, Fisher information computed as in Equation 5 for tone discrimination (black) computed on the basis of frequency response functions (gray dashed) of all frequency-tuned neurons $(n=14)$ recorded in the same mouse as in $\boldsymbol{B}$. $\boldsymbol{D}$, Neurometric threshold for decoding frequency (solid) computed on the basis of the inverse square root of Fisher information computed in $C$. Neurometric threshold based on the recorded population lies above behavioral threshold for discrimination around $f_{1}$ (dashed line). Light blue band indicates the region in frequency space from which behavioral measurements were taken.

\section{Neurometric discrimination thresholds from Fisher information}

Next, we recorded the activity of putative excitatory cells (Materials and Methods) in AC while presenting the awake, head-fixed mouse with a random tone pip stimulus (50 ms tone pips presented every $500 \mathrm{~ms}$, frequency changed at random; Fig. 2A). For each frequency-tuned neuron, we measured frequency response curves (mean firing rate as a function of tone frequency; see Materials and Methods and Fig. 2B). To estimate a discrimination threshold from the frequency-tuned neural population recorded in each mouse, we used Fisher information analysis. To do so, we fit Gaussian functions to the response curve of each neuron and assumed that neurons responded independently and with Poisson variability. Standard methods then provided the Fisher information for frequency discrimination based on the population response (see Eq. 5, Materials and Methods, and Fig. 2C) (Cover and Thomas, 1991). Fisher information quantifies the amount of information that neural responses provide to distinguish nearby frequencies. Decoding sensitivity increases with Fisher information. Because this information is large when the neural response changes quickly as a function of frequency, it is higher on the slopes of the tuning curves than in the center.

The inverse square root of the Fisher information bounds the accuracy with which nearby frequencies can be distinguished based on population activity. This quantity is by definition the neurometric threshold and gives the frequency difference that can be discriminated with $70 \%$ accuracy. Figure $2 D$ shows the neurometric thresholds determined in an individual mouse on the basis of the neural population recorded in its cortex.

A direct comparison of the neurometric threshold based on the limited number of recordings with behavioral performance faces three challenges: (1) discrimination accuracy need not translate linearly into thresholds determined from the PPI of the acoustic startle response measured here, (2) cortical recordings inevitably subsample the population of responsive neurons, and (3) frequency discrimination is supported by multiple pathways, 
some of which may not involve the auditory cortex. In general, we expect the neurometric thresholds computed here to be higher than the behavioral thresholds because of the limited number of recorded neurons. Figure $2 D$ demonstrates such a representative neuron. This discrepancy is likely due to the limited number of neurons and restriction in the brain region in which activity was recorded.

In view of these challenges, we did not seek to predict absolute behavioral thresholds from the population of recorded frequencytuned neurons. Rather, we made a differential estimate: we manipulated excitatory and inhibitory neuronal activity in the auditory cortex optogenetically and tested whether there was a correlation between the resulting changes in the neurometric estimate of discrimination thresholds and corresponding changes in behavior.

\section{Optogenetically induced changes in neurometric and behavioral thresholds are correlated}

Frequency tuning of AC neurons is thought to depend on the combination of excitatory and inhibitory inputs (Chen and Jen, 2000; Wang et al., 2000, 2002; Wehr and Zador, 2003; Aizenberg et al., 2015). To manipulate tone responses of AC neurons, we targeted the most common interneuron subtype, PVs. We drove PVs to express Arch or ChR2 by injecting a floxed Arch- or ChR2encoding virus in PV-Cre mice. We verified the efficiency of the viral transfection and light stimulation by measuring the effect of light on spontaneous firing rates of neurons. As expected, when PVs were activated by light, thus increasing inhibition, spontaneous activity decreased in the recorded population (Fig. 3A) (Natan et al., 2015). Optogenetically suppressing PVs had the opposite effect: the spontaneous rate of most recorded neurons increased (Fig. 3B). We also drove excitatory neurons in AC to express ChR2 and found that optogenetic illumination of ACled to an elevated spontaneous firing rate (Fig. $3 C$ ). Therefore, we reliably used light to manipulate the activity of excitatory and PV neurons in AC.

We next measured the behavioral effects of manipulating neural activity in AC. On half of the trials, we illuminated AC at the same time as providing the prepulse stimulus. Activating PVs (Fig. 3D) decreased the threshold for most animals $(n=5)$ and increased it for some $(n=2)$, whereas a few $(n=4)$ did not have a statistically significant threshold change. Suppressing PVs (Fig. $3 E$ ) produced an increase in the frequency discrimination threshold for two animals and did not produce statistically significant change for one animal. Optogenetically activating excitatory neurons increased the threshold for most animals $(n=3)$ and decreased it for one, with one animal displaying no threshold change (Fig. $3 F)$. For several animals $(n=6)$, the manipulations did not produce any significant behavioral changes.

Finally, we measured the effect of manipulating neuronal activity on the neurometric frequency discrimination thresholds predicted from the recorded population. Activating PVs led to a decrease in the predicted threshold (Figs. 3G,J, 4A, B, blue) for most PV-ChR2 mice $(n=7)$ and an increase for some $(n=2)$. The predicted threshold increased for three PV-Arch mice after suppressing PVs (Figs. $3 \mathrm{H}, \mathrm{K}, 4 \mathrm{~A}, \mathrm{~B}$, green). Activating excitatory neurons (Figs. $3 I, L, 4 A, B$, red) increased the predicted threshold for three CamKIIa-ChR2 mice, but decreased it for two mice. There was no predicted change for two mice $(<2 \%$ change in predicted threshold).

A potential confound for optogenetic manipulation of cortical activity is the scattering of light through the tip of the optic fiber. To prevent the light manipulation to serve as an additional prepulse, we placed a bright LED in the chamber, which served to adapt the retina of the mouse to scattered light from the optic fiber (Danskin et al., 2015). Another limitation of light-driven manipulations of neuronal activity is a potential cascade of prolonged circuit-level dynamics. Therefore, to facilitate the comparison of the effect on light-off and light-on trials, light was presented on light-off trials as well, but not concurrent with the prepulse and startle stimuli. Indeed, light presentation alone did not affect the startle response (PV-ChR2 mice, blue light: $n=11$, $p>0.05$; PV-Arch mice, green light: $n=8, p>0.05)$ and thus did not serve as a prepulse of the acoustic startle response. As an additional control, we injected a cohort of mice with virus carrying only the fluorescent reporter and not the opsin. In these animals, there were no significant effects on frequency discrimination acuity $(n=$ $6, p>0.05$ ) (Aizenberg et al., 2015). These two aspects of stimulus design and control measurements thus confirmed that the observed behavioral changes were unlikely due to artifacts of light presentation, but rather due to light-driven manipulation of cortical activity.

For most individuals $(n=15 / 19)$ and, on average, the sign of the neurometric change in threshold with the optogenetic manipulations matched the sign of the change in behavioral threshold (Fig. 4A,B). This qualitative agreement was striking given that the electrophysiological recordings only sample a few neurons, whereas the light has a global effect on the auditory cortex and sometimes leads to opposite behavioral changes. To quantify the correlation, we compared the neurometric and behavioral frequency discrimination thresholds for each mouse under light-on and light-off conditions. The number of recorded toneresponsive neurons varied significantly between mice (14-104 per animal). Using the linear dependence of the Fisher information on population size, we estimated that $\sim 1000$ independent neurons would be necessary for the neurometric thresholds to match the absolute behavioral thresholds (see Materials and Methods). This number differed between different mice, presumably because of the limited sampling, although was consistent in order of magnitude (Fig. 5). Because optimizing the size of the population for each mouse would have skewed analysis across subjects, we divided the Fisher information computed from the population by the number of neurons and then scaled the result back linearly to the same effective population size for all mice ( $n=1000$ units).

We next compared the resulting estimate to the behavioral threshold. We first found a correlation between the absolute behavioral and neurometric thresholds under all conditions (Fig. 4A, Table 4-1, available at https://doi.org/10.1523/JNEUROSCI. 2457-17.2017.t4-1). The correlation was statistically significant [correlation coefficient $(C)=0.37, p=0.02, n=38$, including a light-on and a light-off measurement for each of 19 mice], but only weakly so. To test more closely the effect of the optogenetic manipulations, we computed an index of change as the difference in thresholds before and after application of light divided by the sum. We found that index of change of the neurometric thresholds was strongly correlated with the behaviorally measured index of change in frequency discrimination acuity threshold $(C=$ $0.59, p=0.007, n=19$; bootstrapped estimates for $C$ : mode $=$ $0.57, \mathrm{SD}=0.1$; Fig. $4 B$ ).

These correlations suggest that: (1) auditory cortex does modulate frequency discrimination behavior, (2) the effects seen in the small recorded patch are representative, and (3) individual differences in auditory behavior are driven directly by differences in excitatory and inhibitory interactions in cortical circuits. 

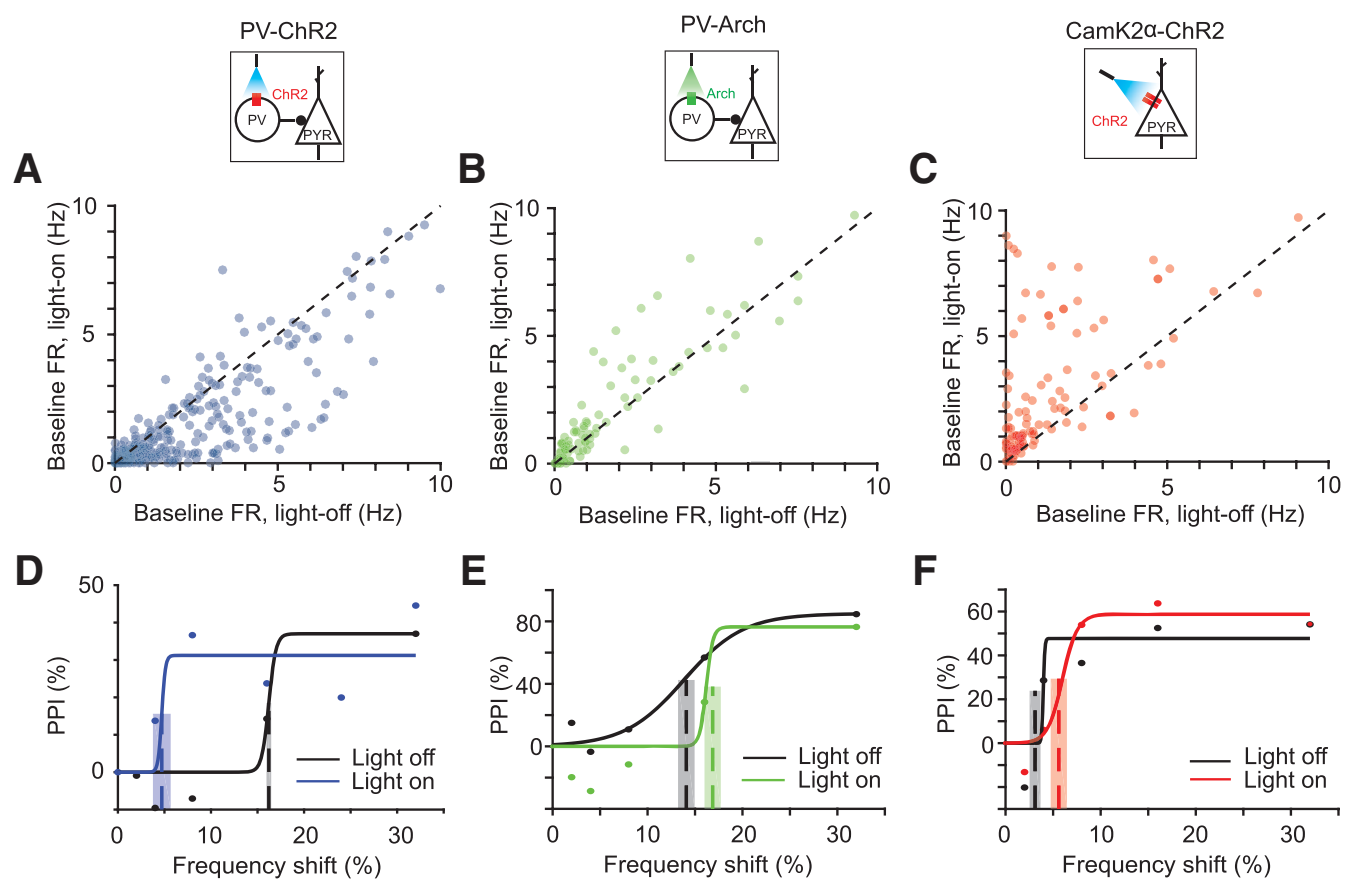

E

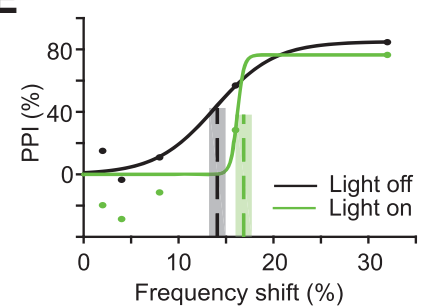

$\mathbf{F}$
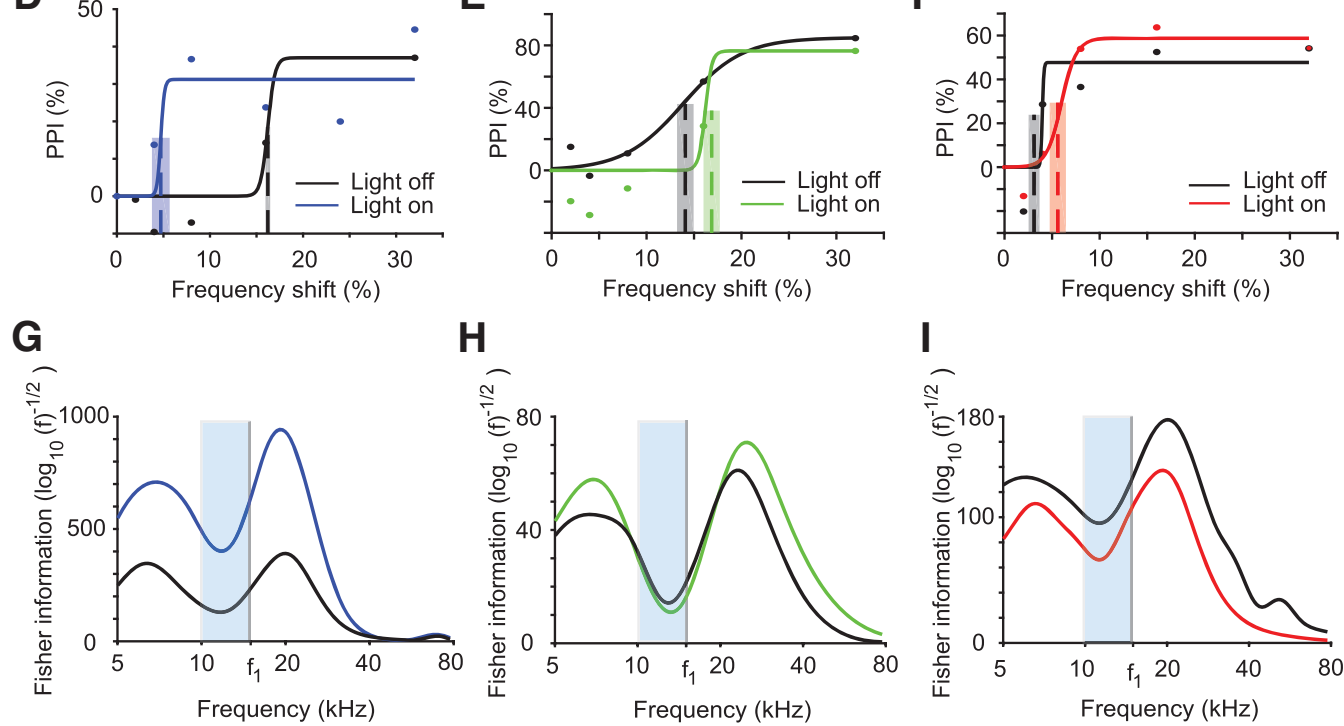

$\mathbf{J}$

\section{K}
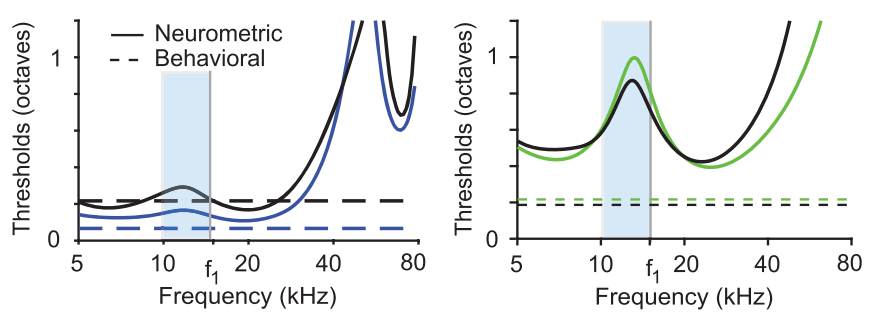

$\mathbf{L}$

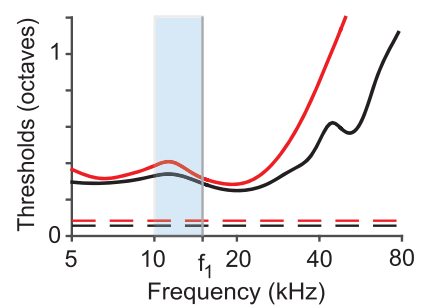

Figure 3. Optogenetic manipulation of PV activity shifts behavioral and neurometric frequency discrimination thresholds in individual subjects. $\boldsymbol{A}-\boldsymbol{C}$, Baseline firing rate of light-on versus light-off trials for all frequency-tuned neurons pooled across subjects in PV-ChR2 (blue), PV-Arch (green), and CamK2a-ChR2 (red) mice, respectively. D- $\boldsymbol{F}$, PPI as a function of tone frequency shift for exemplar mice. Best estimated thresholds (dashed lines) and uncertainties (overlaid gray rectangle) are plotted for reference. Black, Light-off trials; blue, green, red, light-on trials. Dots, Data; solid lines, best fit curve. $\mathbf{G}-\boldsymbol{I}$, Fisher information computed using tuning curves using neurons recorded from mice in $\boldsymbol{D}-\boldsymbol{F}$. Frequencies used are indicated by the blue region. $\boldsymbol{J}-\boldsymbol{L}$, Neurometric threshold estimate as inverse square root of Fisher information (solid) and behavioral threshold at $f_{1}$ (horizontal dotted) for the same mice as $\boldsymbol{D}-\boldsymbol{F}$. Light blue bands indicate the region in frequency space from which behavioral measurements were taken.

\section{Controls: effects of optogenetic manipulation on neural variability and correlations}

Our model makes two assumptions that could be violated in neural systems: that cortical neurons obey Poisson statistics and that neural responses are independent of one another. To test the first assumption in our data, we measured the Fano factor of the recorded neurons. A large Fano factor indicates high neuronal variability (Blackwell et al., 2016). The average Fano factor was around 1.2, similar to the value expected for Poisson neurons and to that previously measured across different cortical areas (Churchland et al., 2010). We found that none of the three optogenetic manipulations $\left(\mathrm{PV}-\mathrm{ChR} 2: t_{(335)}=0.4, p=0.69 ;\right.$ PV-Arch: $t_{(89)}=0.92 ; p=0.36$;
Pyr-ChR2: $t_{(133)}=-0.2 ; p=0.84 ;$ Fig. $\left.7 A-C\right)$ had a systematic effect on the distribution of Fano factors. This justified the original analysis, in which neurons were treated as Poisson both with and without optogenetic manipulation.

We next considered that neural variability has been observed to increase with the activity level of neurons (Goris et al., 2014). It is plausible that the most active neurons, which have the largest impact on Fisher information, may be more variable than expected from the average Fano factor value. The Fisher information decreases as the Fano factor increases (Fig. $6 G$ ) so higher variability in the most active neurons would disproportionately decrease the neurometric discriminability. To test whether this 

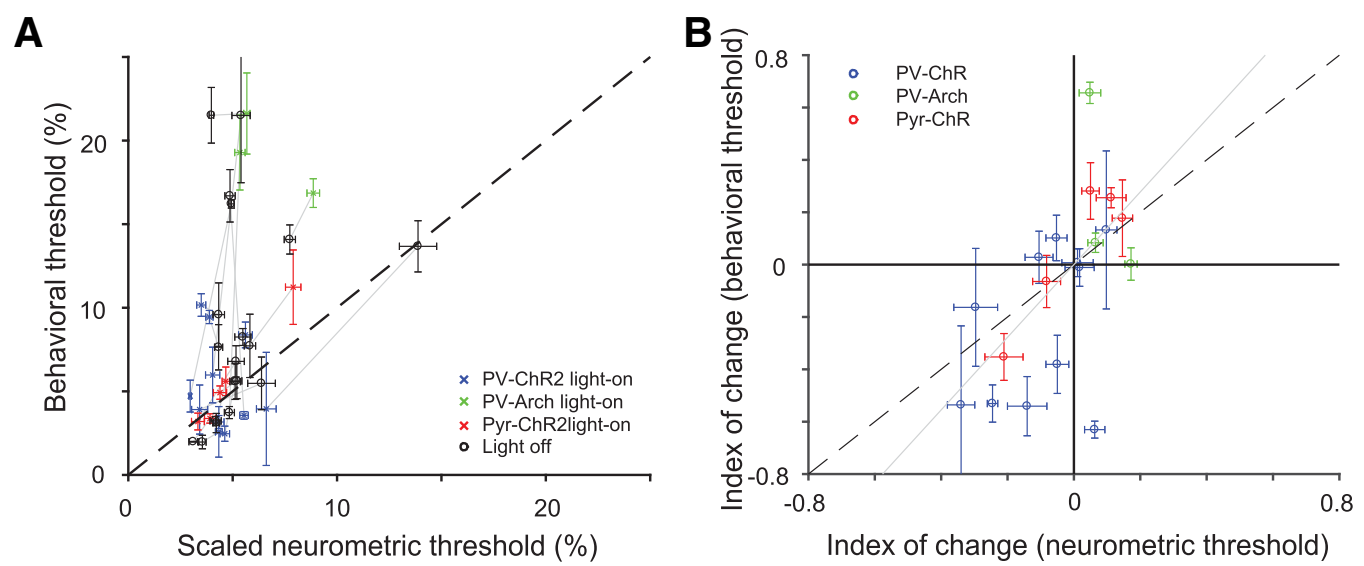

Figure 4. Changes in A1 tone responses due to optogenetic manipulations predict changes in behavioral frequency discrimination acuity across individuals. $\boldsymbol{A}$, Behavioral versus scaled neurometric frequency discrimination thresholds (Table 4-1, available at https://doi.org/10.1523/JNEUROSCI.2457-17.2017.t4-1). Neurometric threshold (computed as inverse of Fisher information squared for tone-evoked responses from all neurons recorded in each mouse) is scaled to an effective population size of 1000 neurons to control for differences in numbers of measured neurons. Changing this scale factor is equivalent to changing $y$-axis labels. The scaled neurometric threshold based on the small recorded population was significantly (but weakly so, $C=0.37, p=0.02$ ) correlated with the behavioral threshold (computed as the shift in frequency between the background and prepulse tone that evoked $50 \%$ of the maximum PPI). Each of 19 mice contributes two data points, representing the threshold computed on the basis of light-on and light-off trials. Gray lines connect light-on and light-off estimates for each mouse. $\boldsymbol{B}$, Index of change in neurometric threshold (difference between thresholds computed from data on light-on vs light-off trials divided by the sum) was strongly correlated with the behavioral frequency discrimination ( $C=0.59$, $p=0.007$ ). There is one data point for each mouse. Gray line is the best fit line through the origin. Behavioral errors were computed as described in the Materials and Methods.

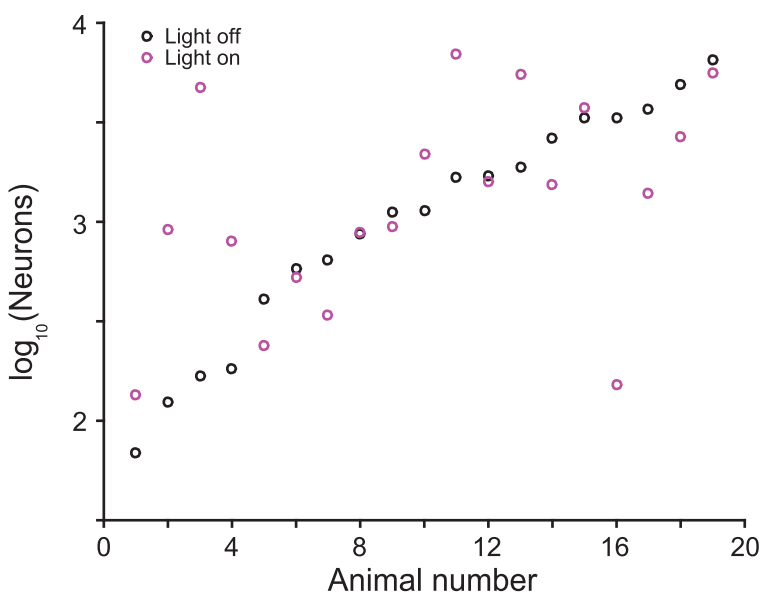

Figure 5. Number of frequency-tuned neurons required to account for behavioral sensitivity for each mouse (Eq. 11). Average of both light-on and light-off conditions is 1000 neurons.

might be the case, we computed the neurometric thresholds using a generalized Poisson noise model that took into account the Fano factor of each recorded neuron separately. Neurometric thresholds using this model changed only slightly from the thresholds computed using the simple Poisson noise model (Fig. $6 \mathrm{H}$ ), validating our assumptions for the original threshold calculation.

We also measured the strength of pairwise neural correlations, and observed they tended to be small but positive $\left(\bar{C}_{P V-C h R 2}=.09\right.$; $t_{(1937)}=28 ; p=4.6^{*} 10^{-141} ; \bar{C}_{P V-\text { Arch }}=.13 ; t_{(524)}=22 ; p=$ $\left.2.2^{\star} 10^{-76} ; \bar{C}_{P y r-C h R 2}=.13 ; t_{(982)}=32 ; p=1.1^{\star} 10^{-155}\right)$. The optogenetic manipulations had no systematic effect on the distribution of correlations (paired $t$ test ns: PV-ChR2, $t_{(1937)}=0.26 ; p=0.80$; PV-Arch, $t_{(524)}=-1.3 ; p=0.18 ;$ Pyr-ChR2, $t_{(982)}=-1.7 ; p=0.09$; Figs. $7 D-F)$. Correlations in similar models have been observed to lead only to small increases in population-level discrimination threshold (Gu et al., 2010). The small changes in threshold observed in previous work, in addition to the negligible change in the correlation distribution that we observed upon optogenetic manipulation, make it unlikely that correlations account for the neurometric threshold changes that we saw when cortex is manipulated.

\section{Discussion}

Cortical neuronal networks consist of dynamically connected populations of excitatory and inhibitory neurons. Theory has shown that providing inputs to inhibitory neurons in a balanced excitatory-inhibitory network can lead to either a decrease or, paradoxically, an increase of excitatory responses, depending on the specifics of recurrent coupling in the network and the effective strength of the manipulation of inhibitory activity (Tsodyks et al., 1997; Levy and Reyes, 2011). By examining changes evoked by optogenetic manipulations in cortical neuronal populations across subjects, we find that modifications of the excitatoryinhibitory interactions in auditory cortex drive diverse, and sometimes opposite, changes in tone-evoked responses across individuals. Remarkably, we found a strong correlation between these changes in neuronal populations and behavioral changes in the acuity of frequency discrimination by individual mice. Therefore, our results demonstrate that the same optogenetic manipulations of excitatory and inhibitory neuronal activity in different individuals can have diverse effects on sensory discrimination. More generally, these findings support a role for excitatory-inhibitory networks in the cortex in mediating sensory discrimination.

From where does this variability in the effects of manipulations across animals arise? Some of the differences may be due to the inherent variability in circuitry across animals: the excitatoryinhibitory circuit can have different connectivity patterns and animals may exhibit differences in frequency sensitivity due to a combination of genetic and environmental factors. In addition, optogenetic manipulations introduce variability across animals due to technical limitations of the technique. In testing the function of specific neuronal cell types in sensory processing, there are a number of potential confounds. For example, the exact position of the needle for virus injection within AC, the depth of penetration, and the spread of the virus can all skew the extent to which the virus is expressed within AC. The position of the optic cannula relative to the spread of the virus injection as well as the 

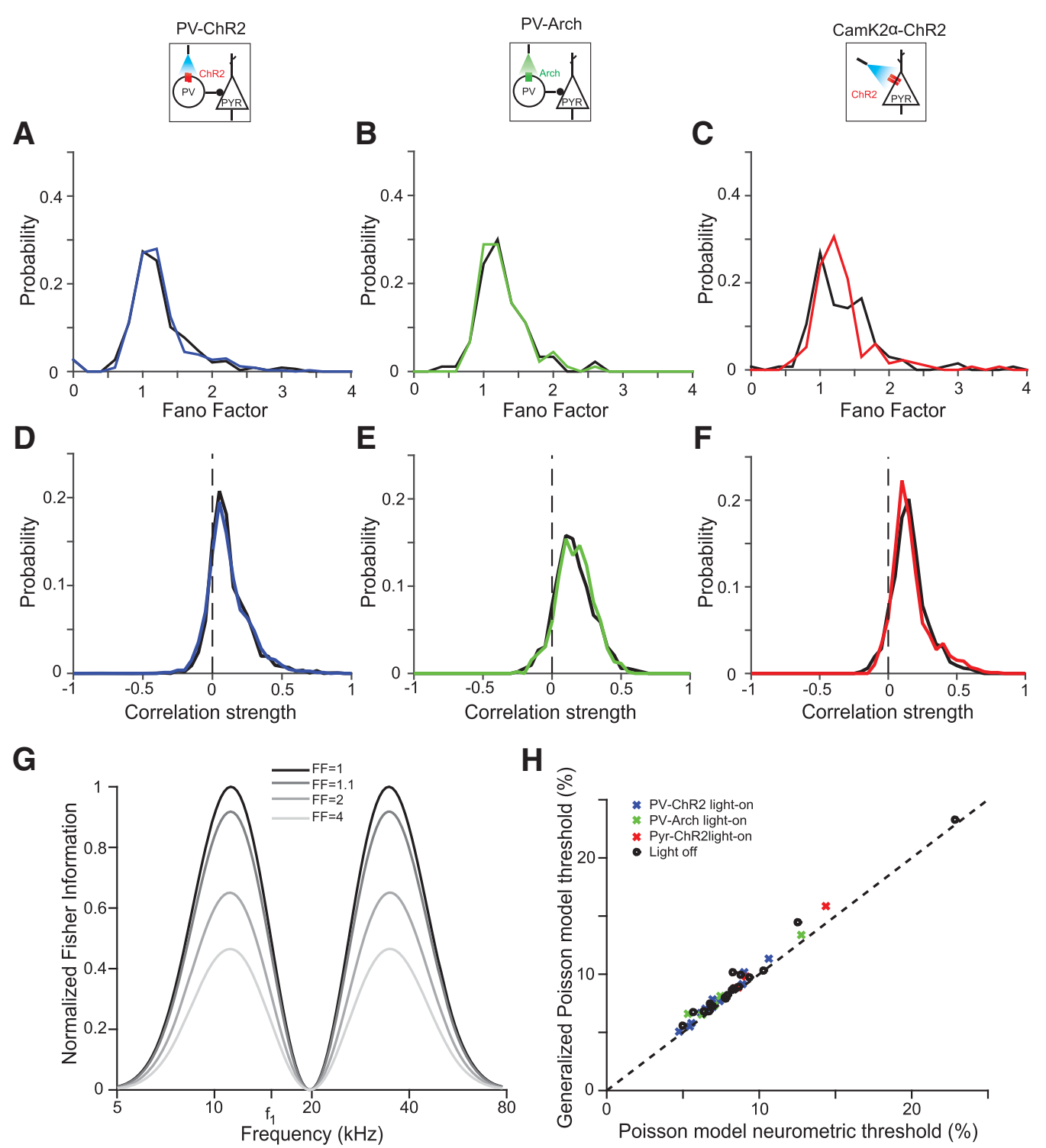

Figure 6. Optogenetic manipulations do not change neuronal variability or correlations. $A-C$, Fano factor pooled across mice distributions are similar under light-on and light-off conditions. $A$, PV-ChR2; $\boldsymbol{B}$, PV-Arch; C, CamK2a-ChR2. Black, Light-off trials; blue, green, red, light-on trials. D-F, Pairwise correlation distributions pooled across mice are similar under light-on and light-off conditions. D, PV-ChR2; $\boldsymbol{E}$, PV-Arch; $\boldsymbol{F}$, CamK2a-ChR2. Colors same as in $\boldsymbol{A}$. G, Increasing Fano factor reduces Fisher information, shown here for a single neuron with Gaussian tuning curve (amplitude 8 spikes/s, center frequency $20 \mathrm{kHZ}$, tuning width 0.2 decades) with a constant baseline ( 2 spikes $/ \mathrm{s})$. $\boldsymbol{H}$, Incorporating the measured Fano factors into our model of neuronal firing via a generalized Poisson model has a weak effect on the predicted threshold.

recording electrode would affect how many neurons are stimulated, how strongly, and how many of those neurons are being picked up by the recording. Small changes in any of these parameters can potentially lead to strong differences in the functional effects of optogenetic perturbations, activating a different fraction of neurons across different laminae and in different tonotopic regions of the cortex. In this study, we capitalized on these differences because they allowed us to assay the range of potential effects of optogenetic manipulations. It was critical, however, to ensure that, within animals, optogenetic manipulation would produce the same effect during behavioral testing and during electrophysiological recordings. We therefore used an implanted optic cannula and a light delivery system with the same settings to deliver light both during the electrophysiological recording and the behavioral measurements so that stimulation parameters were the same for both.

Our results provide insight into potential role of cortical inhibition in shaping frequency discrimination behavior. Cortical inhibitory neurons have been hypothesized to modulate numerous aspects of tone-evoked responses in the excitatory cortical circuit, such as tuning width, reliability of firing, tone-evoked response strength, and correlations in firing rate activity (Wang et al., 2000, 2002; Froemke, 2015). Neurons in the auditory cortex change their tuning properties with learning, attention, or experience (Recanzone et al., 1992; Kilgard and Merzenich, 2002; Fritz et al., 2003; Fritz et al., 2005; Polley et al., 2006; Froemke et al., 2013; Znamenskiy and Zador, 2013), suggesting that these changes can underlie changes in auditory perception. Indeed, AC was shown to play an important function in enabling learning-driven changes in auditory processing (Bajo et al., 2010; Aizenberg and Geffen, 2013). Our present work demonstrates that modulation of excitatory-inhibitory dynamics can, in principle, support a wide range of modulatory effects on auditory frequency discrimination behavior.

The limited size of our recordings of 10-100 frequency-tuned neurons per animal precluded direct prediction of behavioral 


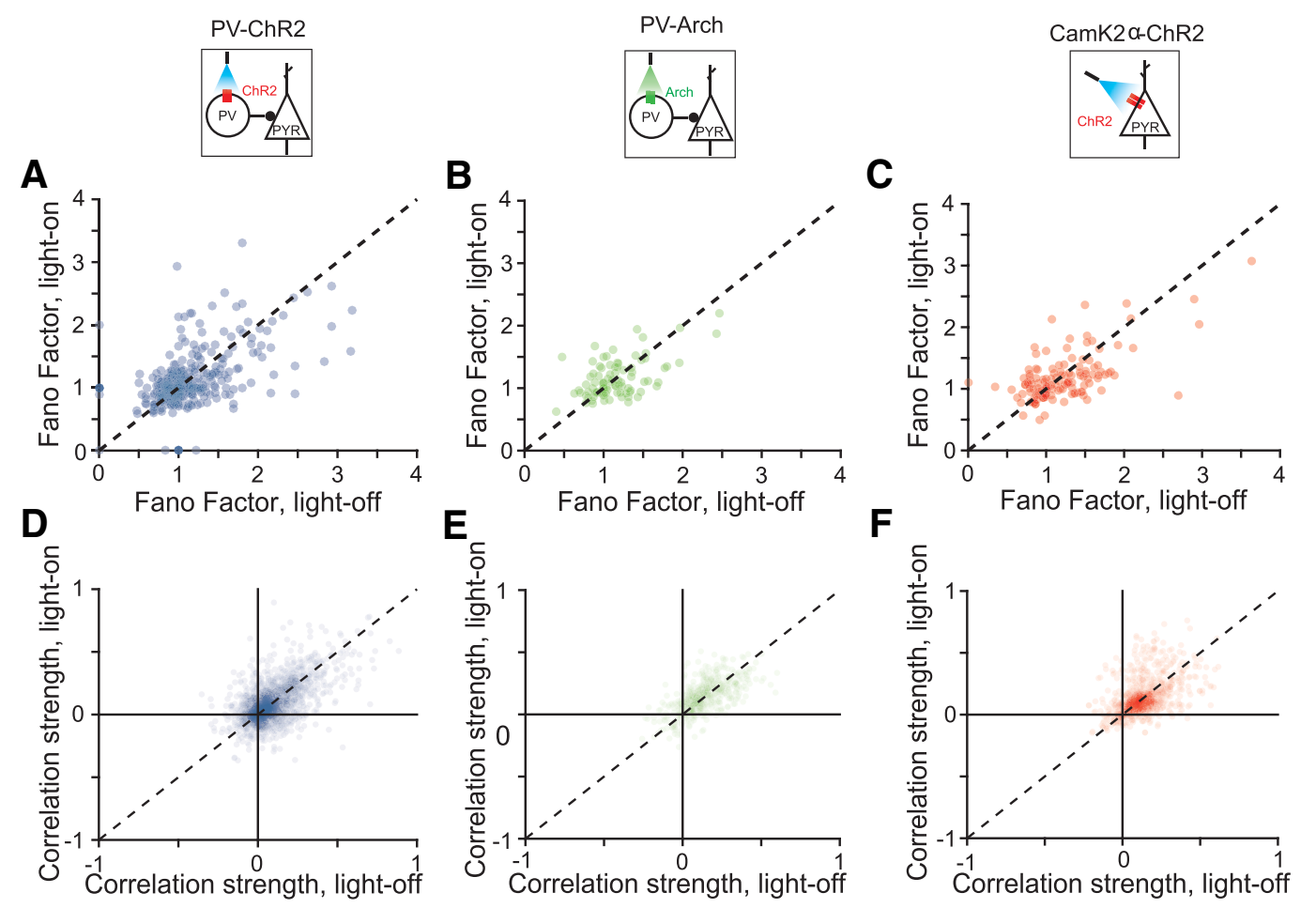

Figure 7. Fano factor and correlation scatter plots comparing light-on and light-off conditions. A-C, Fano factor with and without light on for PV-ChR2, PV-Arch, and Pyr-ChR2 mice, respectively. $D-F$, Pairwise correlations with and without light on for PV-ChR2, PV-Arch, and Pyr-ChR2 mice, respectively.

A
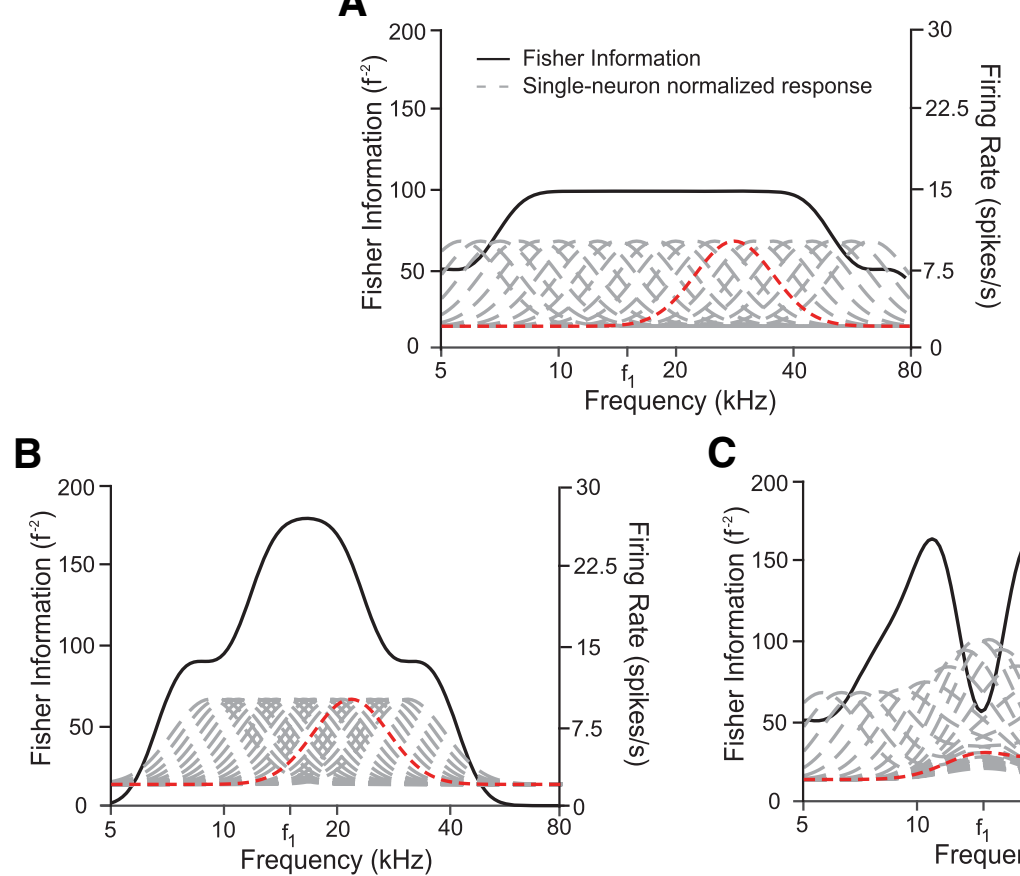

C

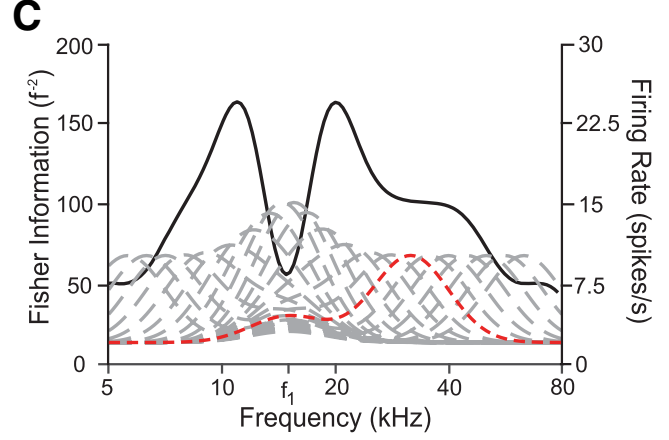

Figure 8. Overrepresenting a specific frequency can increase or reduce sensitivity to that frequency. $A$, Fisher information (black) computed from a homogeneous population of neurons (responses in gray) has an even sensitivity across a broad range of frequencies. A sample tuning curve (red) is used to illustrate neural transformations in $\boldsymbol{B}$ and $\boldsymbol{C}$. Neurons have baseline activity of 2 spikes $/ \mathrm{s}$, peak response of 10 spikes $/ \mathrm{s}$, peak frequency spaced $1 / 20^{\text {th }}$ of a decade apart, with an HWHM of 0.1 decades. $\boldsymbol{B}$, Fisher information is plotted for a neural population overrepresenting frequency $f_{1}$ by shifting peak frequencies halfway between their original location in $A$ and $f_{1}$. Fisher information approximately doubles near $f_{1}$, but is reduced near the edges. $\boldsymbol{C}$, Fisher information is plotted for a neural population overrepresenting frequency $f_{1}$ by adding a Gaussian bump near $f_{1}$ with an amplitude that diminishes with distance between the preferred frequency of the neuron and $f_{1}$. Fisher information is diminished at $f_{1}$, leading to reduced sensitivity at this frequency despite its overrepresentation within the population firing activity.

thresholds: an extrapolation from the measured population indicates that $\mathrm{O}(1000)$ neurons would be required to fully account for behaviorally observed frequency discrimination threshold (Fig. 5). This observation is consistent with an anatomical estimate suggesting that $\mathrm{O}(1000)$ neurons in the mouse $\mathrm{AC}$ are responsive to a given tone [mouse cortex has $\sim 10^{5}$ neurons $/ \mathrm{mm}^{3}$ (Schüz and Palm, 1989), the AC is $\sim 5 \mathrm{~mm}^{3}$ in size, $\sim 30-50 \%$ of neurons are frequency tuned, and the tuning width is $\sim 1 / 10$ of the 
auditory spectrum]. The fact that we were able to make strong predictions about optogenetic effects on behavior despite this subsampling suggests that the changes that we observed in the measured cortical neurons were representative of changes occurring across the entire $\mathrm{AC}$. Comprehensive recordings or imaging of more complete populations of AC neurons will enable a test of the hypothesis that frequency discrimination performance is limited by the encoding at the cortical level.

We observed a relationship between neural responses and behavior under the specific conditions of optogenetic manipulations of cortex, but the methods used here will be broadly useful for understanding other complex phenomena. For example, consider appetitive and aversive conditioning, which have been shown to effect cortical remapping (Bakin and Weinberger, 1990; Recanzone et al., 1993; Polley et al., 2006) while leading to diverse behavioral responses (Aizenberg and Geffen, 2013; Aizenberg et al., 2015). In particular, such remapping leads to an overrepresentation of the aversive stimulus in cortex, but whereas some animals show improved frequency discrimination acuity, others are impaired. In fact, our results are consistent with several models, including overrepresentation of the aversive stimulus that can lead to improved or impaired acuity, depending on the details of the change (Fig. 8). For example, shifting the neural tuning curves toward the aversive frequency leads to increased sensitivity near this tone (Fig. $8 B$ ), whereas simply increasing neural activity in response to tones near the aversive frequency can lead to impaired discrimination (Fig. 8C). The Fisher information analyses that we used have proven generally useful in understanding neural coding, providing insight about sound localization strategies (Harper and McAlpine, 2004), sensitivity to variations in sound levels (Dean et al., 2005), habituation to repeated sounds (Han et al., 2007), detection of sound in noise (Whitton et al., 2014), and heading perception (Gu et al., 2010). Our method allows applications of this technique to compare behavioral discrimination thresholds to neurometric uncertainty in tone frequency estimation.

We were able to discount the effects of inhibition modulation on neuronal variability and correlated activity as driving behavioral changes (Figs. 5, 6). These results differ from theoretical and in vitro predictions for increases neuronal synchronization with increased firing rate due to optogenetic activation (Nassi et al., 2015; Barral and Reyes, 2016). The relatively small effects observed here are likely due to the relatively small amount of perturbation applied to the system through the optogenetic manipulation. Our goal was to keep the system in the physiological regime and the strength of light for manipulations was adjusted to use the smallest power driving a behavioral modulation. It is possible that stronger optogenetic manipulation could drive multiple changes in neuronal synchrony that would affect behavioral discrimination further.

Whereas we used responses to single tones as the stimulus in this study, AC neurons generally respond to more complex sounds in a manner that is not well explained by the single tone responses (Ahrens et al., 2008; Carruthers et al., 2013). Our methods could be used to probe the fidelity of cortical representation and behavior in response to any parameterized space of auditory stimuli; for example, auditory textures, phonemes, or overtone profiles. Similarly to work in visual texture perception (Tkacik et al., 2010; Hermundstad et al., 2014), one could test the discrimination thresholds along different dimensions of the texture space (Geffen et al., 2011; Gervain et al., 2014, 2016). Changes in these thresholds due to optogenetic circuit manipulation could then be compared with changes in a neurometric threshold based on Fisher information, as applied here. Such studies will elucidate the role of AC in facilitating complex auditory discrimination.

The analysis presented here agrees with and clarifies our previous results (Aizenberg et al., 2015), in which we reported that activating $\mathrm{PV}$ on average increased neuronal response strength in $\mathrm{AC}$ and improved frequency discrimination acuity, whereas suppressing PVs decreased response strength and impaired acuity. Here, we provide additional evidence for the link between cortical activity and behavioral discrimination on an animal-byanimal basis, confirming the result that the auditory cortex modulates frequency discrimination acuity. By examining individual differences, it becomes clear that the effects of optogenetic manipulations are more nuanced than can be understood by examining the average effects: the same manipulation can drive changes on individual basis in opposite direction in both neuronal responses and behavior and there are numerous explanations that could account for these changes. Indeed, rather than serving a predefined static function, excitatory-inhibitory connections can support a number of behaviorally and experience-driven modulation trajectories, giving rise to adaptive behavior. We suggest that such attention to individual variation will be broadly important throughout neuroscience as large-scale recordings begin to reveal the neural basis of behavioral diversity.

\section{References}

Ahrens MB, Linden JF, Sahani M (2008) Nonlinearities and contextual influences in auditory cortical responses modeled with multilinear spectrotemporal methods. J Neurosci 28:1929-1942. CrossRef Medline

Aizenberg M, Geffen MN (2013) Bidirectional effects of auditory aversive learning on sensory acuity are mediated by the auditory cortex. Nat Neurosci 16:994-996. CrossRef Medline

Aizenberg M, Mwilambwe-Tshilobo L, Briguglio JJ, Natan RG, Geffen MN (2015) Bidirectional regulation of innate and learned behaviors that rely on frequency discrimination by cortical inhibitory neurons. PLoS Biol 13:e1002308. CrossRef Medline

Atallah BV, Bruns W, Carandini M, Scanziani M (2012) Parvalbuminexpressing interneurons linearly transform cortical responses to visual stimuli. Neuron 73:159-170. CrossRef Medline

Atasoy D, Aponte Y, Su HH, Sternson SM (2008) A FLEX switch targets channelrhodopsin-2 to multiple cell types for imaging and long-range circuit mapping. J Neurosci 28:7025-7030. CrossRef Medline

Bajo VM, Nodal FR, Moore DR, King AJ (2010) The descending corticocollicular pathway mediates learning-induced auditory plasticity. Nat Neurosci 13:253-260. CrossRef Medline

Bakin JS, Weinberger NM (1990) Classical conditioning induces CSspecific receptive field plasticity in the auditory cortex of the guinea pig. Brain Res 536:271-286. CrossRef Medline

Barral J, A DR (2016) Synaptic scaling rule preserves excitatory-inhibitory balance and salient neuronal network dynamics. Nat Neurosci 19:16901696. CrossRef Medline

Blackwell JM, Taillefumier TO, Natan RG, Carruthers IM, Magnasco MO, Geffen MN (2016) Stable encoding of sounds over a broad range of statistical parameters in the auditory cortex. Eur J Neurosci 43:751-764. CrossRef Medline

Boyden ES, Zhang F, Bamberg E, Nagel G, Deisseroth K (2005) Millisecondtimescale, genetically targeted optical control of neural activity. Nat Neurosci 8:1263-1268. CrossRef Medline

Cardin JA, Carlén M, Meletis K, Knoblich U, Zhang F, Deisseroth K, Tsai LH, Moore CI (2010) Targeted optogenetic stimulation and recording of neurons in vivo using cell-type-specific expression of channelrhodopsin-2. Nat Protoc 5:247-254. CrossRef Medline

Carruthers IM, Natan RG, Geffen MN (2013) Encoding of ultrasonic vocalizations in the auditory cortex. J Neurophysiol 109:1912-1927. CrossRef Medline

Chen QC, Jen PH (2000) Bicuculline application affects discharge patterns, rate-intensity functions, and frequency tuning characteristics of bat auditory cortical neurons. Hear Res 150:161-174. CrossRef Medline

Chow BY, Han X, Dobry AS, Qian X, Chuong AS, Li M, Henninger MA, Belfort GM, Lin Y, Monahan PE, Boyden ES (2010) High-performance 
genetically targetable optical neural silencing by light-driven proton pumps. Nature 463:98-102. CrossRef Medline

Churchland MM, et al. (2010) Stimulus onset quenches neural variability: a widespread cortical phenomenon. Nat Neurosci 13:369-378. CrossRef Medline

Clause A, Nguyen T, Kandler K (2011) An acoustic startle-based method of assessing frequency discrimination in mice. J Neurosci Methods 200:6367. CrossRef Medline

Cover TM, Thomas JA (1991) Elements of information theory. New York: Wiley.

Danskin B, Denman D, Valley M, Ollerenshaw D, Williams D, Groblewski P, Reid C, Olsen S, Blanche T, Waters J (2015) Optogenetics in mice performing a visual discrimination task: measurement and suppression of retinal activation and the resulting behavioral artifact. PLoS One 10: e0144760. CrossRef Medline

Dean I, Harper NS, McAlpine D (2005) Neural population coding of sound level adapts to stimulus statistics. Nat Neurosci 8:1684-1689. CrossRef Medline

Deisseroth K (2011) Optogenetics. Nat Methods 8:26-29. CrossRef Medline

Erway LC, Willott JF, Archer JR, Harrison DE (1993) Genetics of age-related hearing loss in mice: I. Inbred and F1 hybrid strains. Hear Res 65:125-132.

Fritz JB, Elhilali M, Shamma SA (2005) Differential dynamic plasticity of A1 receptive fields during multiple spectral tasks. J Neurosci 25:7623-7635. CrossRef Medline

Fritz J, Shamma S, Elhilali M, Klein D (2003) Rapid task-related plasticity of spectrotemporal receptive fields in primary auditory cortex. Nat Neurosci 6:1216-1223. CrossRef Medline

Froemke RC (2015) Plasticity of cortical excitatory-inhibitory balance. Annu Rev Neurosci 38:195-219. CrossRef Medline

Froemke RC, Carcea I, Barker AJ, Yuan K, Seybold BA, Martins AR, Zaika N, Bernstein H, Wachs M, Levis PA, Polley DB, Merzenich MM, Schreiner CE (2013) Long-term modification of cortical synapses improves sensory perception. Nat Neurosci 16:79-88. CrossRef Medline

Geffen MN, Gervain J, Werker JF, Magnasco MO (2011) Auditory perception of self-similarity in water sounds. Front Integr Neurosci 5:15. CrossRef Medline

Gervain J, Werker JF, Geffen MN (2014) Category-specific processing of scale-invariant sounds in infancy. PLoS One 9:e96278. CrossRef Medline

Gervain J, Werker JF, Black A, Geffen MN (2016) The neural correlates of processing scale-invariant environmental sounds at birth. Neuroimage 133:144-150. CrossRef Medline

Goris RL, Movshon JA, Simoncelli EP (2014) Partitioning neuronal variability. Nat Neurosci 17:858-865. CrossRef Medline

Gu Y, Fetsch CR, Adeyemo B, Deangelis GC, Angelaki DE (2010) Decoding of MSTd population activity accounts for variations in the precision of heading perception. Neuron 66:596-609. CrossRef Medline

Han YK, Köver H, Insanally MN, Semerdjian JH, Bao S (2007) Early experience impairs perceptual discrimination. Nat Neurosci 10:1191-1197. CrossRef Medline

Harper NS, McAlpine D (2004) Optimal neural population coding of an auditory spatial cue. Nature 430:682-686. CrossRef Medline

Hermundstad AM, Briguglio JJ, Conte MM, Victor JD, Balasubramanian V, Tkacik G (2014) Variance predicts salience in central sensory processing. Elife 3.

Isaacson JS, Scanziani M (2011) How inhibition shapes cortical activity. Neuron 72:231-243. CrossRef Medline

Johnson KR, Tian C, Gagnon LH, Jiang H, Ding D, Salvi R (2017) Effects of Cdh23 single nucleotide substitutions on age-related hearing loss in C57BL/6 and 129S1/Sv mice and comparisons with congenic strains. Scientific reports 7:44450.

Kilgard MP, Merzenich MM (2002) Order-sensitive plasticity in adult primary auditory cortex. Proc Natl Acad Sci U S A 99:3205-3209. CrossRef Medline

Lee SH, Kwan AC, Zhang S, Phoumthipphavong V, Flannery JG, Masmanidis SC, Taniguchi H, Huang ZJ, Zhang F, Boyden ES, Deisseroth K, Dan Y (2012) Activation of specific interneurons improves V1 feature selectivity and visual perception. Nature 488:379-383. CrossRef Medline
Levy RB, Reyes AD (2011) Coexistence of lateral and co-tuned inhibitory configurations in cortical networks. PLoS Comput Biol 7:e1002161. CrossRef

Marlin BJ, Mitre M, D’amour JA, Chao MV, Froemke RC (2015) Oxytocin enables maternal behaviour by balancing cortical inhibition. Nature 520 : 499-504. CrossRef Medline

Mikaelian DO (1979) Development and degeneration of hearing in the C57/ b16 mouse: relation of electrophysiologic responses from the round window and cochlear nucleus to cochlear anatomy and behavioral responses. The Laryngoscope 89:1-15. CrossRef Medline

Mwilambwe-Tshilobo L, Davis AJ, Aizenberg M, Geffen MN (2015) Selective impairment in frequency discrimination in a mouse model of tinnitus. PLoS One 10:e137749. CrossRef Medline

Nassi JJ, Avery MC, Cetin AH, Roe AW, Reynolds JH (2015) Optogenetic Activation of Normalization in Alert Macaque Visual Cortex. Neuron 86:1504-1517. CrossRef Medline

Natan RG, Briguglio JJ, Mwilambwe-Tshilobo L, Jones SI, Aizenberg M, Goldberg EM, Geffen MN (2015) Complementary control of sensory adaptation by two types of cortical interneurons. eLife 4: pii: e09868. CrossRef Medline

Phillips EA, Hasenstaub AR (2016) Asymmetric effects of activating and inactivating cortical interneurons. Elife 5. pii: e18383. CrossRef Medline

Polley DB, Steinberg EE, Merzenich MM (2006) Perceptual learning directs auditory cortical map reorganization through top-down influences. J Neurosci 26:4970-4982. CrossRef Medline

Recanzone GH, Merzenich MM, Schreiner CE (1992) Changes in the distributed temporal response properties of SI cortical neurons reflect improvements in performance on a temporally based tactile discrimination task. J Neurophysiol 67:1071-1091. CrossRef Medline

Recanzone GH, Schreiner CE, Merzenich MM (1993) Plasticity in the frequency representation of primary auditory cortex following discrimination training in adult owl monkeys. J Neurosci 13:87-103. Medline

Schüz A, Palm G (1989) Density of neurons and synapses in the cerebral cortex of the mouse. J Comp Neurol 286:442-455. CrossRef Medline

Seybold BA, Phillips EAK, Schreiner CE, Hasenstaub AR (2015) Inhibitory actions unified by network integration. Neuron 87:1181-1192. CrossRef Medline

Sohal VS, Zhang F, Yizhar O, Deisseroth K (2009) Parvalbumin neurons and gamma rhythms enhance cortical circuit performance. Nature 459: 698-702. CrossRef Medline

Tkacik G, Prentice JS, Victor JD, Balasubramanian V (2010) Local statistics in natural scenes predict the saliency of synthetic textures. Proc Natl Acad Sci U S A 107:18149-18154. CrossRef Medline

Tsodyks MV, Skaggs WE, Sejnowski TJ, McNaughton BL (1997) Paradoxical effects of external modulation of inhibitory interneurons. J Neurosci 17:4382-4388. Medline

Wang J, Caspary D, Salvi RJ (2000) GABA-A antagonist causes dramatic expansion of tuning in primary auditory cortex. Neuroreport 11:11371140. CrossRef Medline

Wang J, McFadden SL, Caspary D, Salvi R (2002) Gamma-aminobutyric acid circuits shape response properties of auditory cortex neurons. Brain Res 944:219-231. CrossRef Medline

Wehr M, Zador AM (2003) Balanced inhibition underlies tuning and sharpens spike timing in auditory cortex. Nature 426:442-446. CrossRef Medline

Whitton JP, Hancock KE, Polley DB (2014) Immersive audiomotor game play enhances neural and perceptual salience of weak signals in noise. Proc Natl Acad Sci U S A 111:E2606-E2615. CrossRef Medline

Wilson NR, Runyan CA, Wang FL, Sur M (2012) Division and subtraction by distinct cortical inhibitory networks in vivo. Nature 488:343-348. CrossRef Medline

Zhang F, Wang LP, Brauner M, Liewald JF, Kay K, Watzke N, Wood PG, Bamberg E, Nagel G, Gottschalk A, Deisseroth K (2007) Multimodal fast optical interrogation of neural circuitry. Nature 446:633-639. CrossRef Medline

Znamenskiy P, Zador AM (2013) Corticostriatal neurons in auditory cortex drive decisions during auditory discrimination. Nature 497:482-485. CrossRef Medline 